\title{
Improving the Registration Process in a Healthcare Facility with Lean Principles
}

\author{
Valentina Nino $^{1}$ (D), Kenneth J. Martínez ${ }^{1}$ (D), Karina Gómez ${ }^{2}$ (D), David Claudio ${ }^{1}$ iD \\ ${ }^{1}$ Department of Mechanical and Industrial Engineering, Montana State University, Bozeman, MT (United States) \\ ${ }^{2}$ Universidad de Puerto Rico, Mayaguez, (Puerto Rico) \\ valentinanino@gmail.com,k.j_mart_@rocketmail.com,karina.gomez4@upr.edu,david.claudio@montana.edu
}

Received: December 2020

Accepted: May 2021

\begin{abstract}
:
Purpose: Process improvement in service industries, like the registration process in a hospital, can be achieved with the application of lean principles. In this specific case, lean principles were essential to analyze and improve patient satisfaction in a hospital in Montana. The clinics involved in the study included pediatrics (P), internal medicine (IM), and cardiology/pulmonary (CP). The purpose of this study was to address difficulties regarding patient satisfaction on the registration and check-in processes.
\end{abstract}

Design/methodology/approach: Direct observations and patient surveys were initially made to understand the processes and identify the initial causes of dissatisfaction. A value stream map (VSM) was then used to further break down the patient flow's complexity. A Fishbone diagram and a Concept Map were completed to find the root of the dissatisfaction.

Findings: The information obtained from the lean tools showed that patients questioned the need for having a central registration in combination with the check-in process when this combination generates duplications of steps that cause unwanted delays. Several recommendations were explored by the engineering team to mitigate these delays and improve the registration process reducing the number of patients complaints by $40 \%$.

Practical implications: This project illustrated the application of lean principles to resolve issues regarding a central registration format in a healthcare facility.

Originality/value: A Concept Map was used as a tool to help the organization develop creative and new ways of looking at and solving process deficiencies.

Keywords: lean principles, patient satisfaction, quality improvement, central registration

\section{To cite this article:}

Nino, V., Martínez, K.J., Gómez, K., \& Claudio, D. (2021). Improving the registration process in a healthcare facility with lean principles. Journal of Industrial Engineering and Management, 14(3), 538-551.

https://doi.org/10.3926/jiem.3432 


\section{Introduction}

Patient satisfaction can be significantly jeopardized by any process or element in the care delivery stream. It is a critical service quality indicator that can signal opportunities for improvement in a hospital setting (Yellen, Davis \& Ricard, 2002).

Since 2008, the United States has implemented the Hospital Consumer Assessment of Healthcare Providers and Systems (HCAHPS) as a standard comparison tool for satisfaction criteria collection and reporting (Giordano, Elliott, Goldstein, Lehrman \& Spencer, 2010; Wild, Kwon, Dutta, Tessier-Sherman, Woddor, Sipsma et al., 2011). It allows patients to compare healthcare facilities' performance based on 32 criteria that are critical to the patient. HCAHPS reports on the satisfaction levels to the public and accreditation through the Centers for Medicare and Medicaid Services (CMS). CMS ensures that healthcare facilities meet specified guidelines in the provider's operations and performance. This relates to potential reimbursement for higher satisfaction ratings for high-performing clinics.

HCAHPS was created with the intent of reshaping and conceptualizing patient satisfaction in healthcare. It incorporates several predictors such as staff responsiveness, data management, medication delivery, discharge process, and physical environment (Mazurenko, Collum, Ferdinand \& Menachemi, 2017). Aspects such as service accessibility and the physical environment have been identified as highly influential in measuring patient satisfaction (Batbaatar, Dorjdagva, Luvsannyam, Savino \& Amenta, 2017; MacAllister, Zimring \& Ryherd, 2016; Meesala \& Paul, 2018; Suess \& Mody, 2018).

Accessibility is an aspect within the physical environment that plays a significant role in service quality (Deshmukh, Seth \& Vrat, 2005; Oliver, 1993; Parasuraman, Zeithaml \& Berry, 1985). It can be influenced directly by how elements are arranged or placed in a facility. Therefore, engineers and designers need to be aware of how their designs are addressable to the whole public and include special self-learnable features to enable full usability and functionality (Martin-Escalona, Barcelo-Arroyo \& Zola, 2013). Facility design juxtaposed with operational models can produce outcomes that improve patient experience (Jha, Frye, \& Schlimgen, 2017). To this goal, Lean thinking and Lean principles can help remove unnecessary motion, waiting, and other non-value-added activities by streamlining processes (Wickramasinghe, 2014).

Many healthcare systems exhibit inappropriate features, inflexibility, no error prevention mechanisms, and documentation issues (Dias, Pereira, \& Freire, 2017). Situations like these generate confusion and delay care, prompting a great assortment of complaints from unsatisfied patients. Lean principles have been recited and introduced as a series of steps for successful accomplishment of improvements in healthcare facilities (Daultani, Chaudhuri \& Kumar, 2015; Gershenson \& Pavnaskar, 2003; Hicks, McGovern, Prior \& Smith, 2015; Machado \& Leitner, 2010; Series, 2005; Spagnol, Min \& Newbold, 2013; Womack, Jones, Roos, 1990) and optimization of patient flow (Leviner, 2020). Daultani et al. (2015) reference various projects that had claimed success in healthcare settings through Lean implementation. Reported benefits include simplified processes (i.e., reduced number of steps), reduced turnaround time and scheduling confusions, decreased number of safety incidents, lower waiting times, financial savings, and optimized facility design for staff and patients.

Many researchers have suggested applying lean thinking to assess the design and delivery of health services (Abdu, Peter \& Gupta, 2016; Daultani et al., 2015; Gershenson \& Pavnaskar, 2003; Hallam \& Contreras, 2018; Hicks et al., 2015; Kadarova \& Demecko, 2016; Sobek, 2011; Solaimani, van der Veen, Sobek, Gulyaz \& Venugopal, 2019). Lot, Sarantopoulos, Min, Perales, Boin and Ataide (2018) took direct observations from a Gemba walk to understand how the process worked in a public clinic and developed a value stream map (VSM) to suggest where waiting times could be reduced. Faulkner (2013) used process mapping to draw the current state and identify value and wastes in a postpartum unit. Faulkner's team utilized a fishbone diagram and 5-Whys technique to uncover significant issues that stalled quick and efficient responses to postpartum hemorrhage cases. Other studies have addressed patient satisfaction in healthcare on a general level (Faezipour \& Ferreira, 2013; Fatima, Malik Shahab \& Shabbir, 2018; Mathew, Narayanan \& Mittal, 2019; Moretta-Tartaglione, Cavacece, Cassia \& Russo, 2018).

This study focused on the central registration (CR) process in a Montana healthcare facility and explored how perception ultimately affected patients' experience and, therefore, customer service. Initially, the registration and 
check-in processes occurred in the clinic that patients used to attend. However, the hospital administration started having problems with the documentation of patients to bill health insurance companies. Likewise, the hospital continued expanding. In an attempt to improve staff efficiency and reduce costs, a new building was designed with a central registration area and smaller check-in spaces at the clinics. The idea behind it all was that the clinics would take care of the healthcare services required by the patients. The central registration (CR) would manage the health insurance information and billing documentation.

The CR in this facility is in charge of registering all patients served by the clinics located in the hospital, merging those individualized services into one. This way, the quality of the information collected would be improved in terms of completeness, consistency, and accuracy. In an initial stage, the hospital's managers decided to consolidate the registration services from three of its clinics: internal medicine (IM), pediatric (P), and cardiology/pulmonary (CP) happening now in the CR area. This new arrangement of the registration and check-in processes had negative effects on patient satisfaction. Since January 2018, the Montana hospital has had overwhelming complaints concerning patient check-in and registration logistics. The primary complaint was that CR was unintentionally bypassed and skipped by patients.

This study used lean tools to identify what became dissatisfying for patients at the Montana healthcare facility and how to systematically approach patient experience through lean principles. The five principles of lean-define value, map value stream, create flow, establish pull, and pursue continuous improvement (Womack et al., 1990) -served as the baseline to improve the central registration process. Protocols and physical elements related to the CR process were assessed. The array of CR complaints was the starting point and proved suitable for the intervention made with the aforementioned lean principles and tools in this scenario.

A Concept Map was used to help the organization develop creative new ways of looking at and solving process deficiencies. In recent years, Concept Maps have expanded beyond the field of education to the business world as a powerful tool to present information based on meaningful connections (Anderson, Day \& Vandenberg, 2011; Egelhoff, Podoll \& Tarhini, 2010). In our case, using the Concept Map helped the team in modeling and identifying complex relationships among areas and processes, mapping team knowledge, and spotting gaps and contradictions in the observed processes.

The remainder of this document is organized as follows: Section two provides a description of the methods and tools used in each of the five lean principles followed to addresses the issue. Section three details the information collected, and the results obtained at each step of the study. Section four discusses the implications of the results. Finally, section five summarizes the findings along with the benefits of systematically addressing and solving problems through the five principles of lean.

\section{Methods}

The study's hospital is a rural accredited Level-3 trauma center located in Montana, USA. The hospital provides care in approximately 35 specialties.

The process improvement project presented here followed the five principles of lean; define value, map value steam, create flow, establish pull, and pursue continuous improvement (Womack et al. 1990). The first step was to define value for which direct observations of the CR process were conducted. Natural patients' flow from their arrival to the check-out process was carefully observed. Time studies of the registration process, the time spent for patients to move from CR to their respective clinic, and check-in processes in the three clinics (IM, P, CP) were conducted to observe and gather information about the time spent by the patients in the system and waiting times. Time studies were conducted for a period of two weeks. Ten days of direct data were collected from the CR process and IM, P, and CP clinics assuring all shifts were included in the study. It was possible to have a complete picture of the processes, including peak hours and times where the number of patients visits were low. Furthermore, to hear patients' opinions about the process and to identify what was important from the customer's point of view, patients were surveyed concerning the matter. From January 2018 to March 2019, the hospital had 61,057 visits in the studied clinics. A total of 4,506 impressions were collected. 
The second step was to map the value stream. A value stream map (VSM) that navigates from the registration process to check-out was created to better understand the entire service cycle. The VSM was completed from direct observations and constant discussions between hospital stakeholders. Stakeholders included clinics and CR managers, administrative staff, medical staff such as nurses and physicians, the hospital quality improvement department manager and staff, and the engineering team integrated of Industrial Engineering students and their advisor.

A concept map was then prepared by the engineering team to evaluate the current state of the CR process. Concept maps are broadly used in education settings because they effectively produce visual representations of information and knowledge. They show concepts and ideas and the relationship between them. A concept map is a user-friendly, intuitive, easy-to-do, highly visual communicator, and storytelling tool that helps organizations develop new creative ways of looking and solving process deficiencies (Anderson et al., 2011; Egelhoff et al., 2010). Concept mapping is, by extension, a group process that makes the tool an ideal one for groups of stakeholders working together when solving problems.

Once the process was mapped, a fishbone diagram was created to track down the possible reasons for errors within the process(es). A cause-and-effect analysis (i.e., Fishbone diagram) allowed the engineering team to deeply appreciate the problem and break down the hows, wheres, and when. This diagram was prepared with the constant feedback of hospital stakeholders. The results and insights collected from applying the tools mentioned above were analyzed through group discussions, presentations, and brainstorming sessions, allowing the team (hospital stakeholders and engineers) to create flow and establish pull defining how the hospital wanted to perform the registration check-in processes. Finally, hospital stakeholders, along with the engineering team were able to propose the improvement recommendations. This step was accomplished through discussions and brainstorming sessions.

\section{Results}

\subsection{Define Value}

The first step was to define value; therefore, a survey was applied to patients to identifying what was important from the customer's point of view concerning the CR process; approximately 4,500 impressions were collected. The number of negative comments gathered about CR were classified into four categories: purpose redundancy, privacy, wayfinding distance, and wait time (Table 1).

\begin{tabular}{|c|c|c|c|c|c|c|c|c|}
\hline \multirow[b]{2}{*}{ Patient destination } & \multicolumn{4}{|c|}{ Complaint Category } & \multirow[b]{2}{*}{ Total } & \multirow[b]{2}{*}{$\begin{array}{l}\text { Total } \\
\text { Visits }\end{array}$} & \multirow[b]{2}{*}{$\begin{array}{c}\text { Survey } \\
\text { Returned }\end{array}$} & \multirow[b]{2}{*}{$\%$} \\
\hline & $\begin{array}{c}\text { Purpose } \\
\text { Redundancy }\end{array}$ & Privacy & $\begin{array}{l}\text { Wayfinding } \\
\text { Distance }\end{array}$ & $\begin{array}{l}\text { Wait } \\
\text { time }\end{array}$ & & & & \\
\hline Internal Medicine & 40 & 6 & 14 & 4 & 64 & 37381 & 3940 & 10.54 \\
\hline Pediatric & 2 & 1 & 0 & 5 & 8 & 21739 & 454 & 2.09 \\
\hline Cardiology/Pulmonary & 0 & 1 & 0 & 0 & 1 & 1937 & 112 & 5.78 \\
\hline Total & 42 & 8 & 14 & 9 & 73 & 61057 & 4506 & 1.38 \\
\hline
\end{tabular}

Table 1. Matrix of complaints about central registration (CR)

After the revision of the 4,506 surveys, only 73 of them had to do with either the process itself or facility issues. The top two categories were purpose redundancy $(57.5 \%)$ and wayfinding distance $(19.2 \%)$ that had created unwanted variability in the process, which is the counterpart of a lean process, deriving from a lack of consistency and unexpected service interruptions.

Redundancy refers here to a situation in which a patient perceives doing the same task over again similar to overprocessing. In healthcare terms, overprocessing could occur upon the mere duplication of steps, such as asking a patient for details several times or ask them to go to the same process more than once (Robinson, Radnor, Burgess \& Worthington, 2012). This source of waste can happen when patients are obligated to travel the same distances again and again. For instance, in our case, a patient was seen first by a front-desk staff member at the CR 
area who eventually sent the patient up to the clinics to be checked in. By doing so, the patient ends up seeing two or three front-desk staff members (when patients skip registration); therefore, repeated steps or overprocessing became an evident issue.

Regarding wayfinding, in our study, patients were losing track of where they needed to go and where exactly the place was located. Wayfinding has been considered by many researchers as a challenge left unchanged for years. Maqbool, Raju and In (2016) specify from experience that poor, unclear signage and instructions can result in ineffective navigation from and to a unit. When changes in a facility have recently happened, it is imperative even for staff to trust wayfinding resources such as signs, maps, and floor graphics. However, some of those wayfinding resources present shortcomings and complications, yielding undesired uncertainties and delays in a process (Nunnally \& Bitan, 2014).

\subsection{Map the Value Stream}

The next step was mapping the process by using a Value Stream Map (VSM). From direct observations and constant discussions with hospital stakeholders, the VSM that navigates from the registration process to certain check-outs was elaborated to better understand how patients go through the full process (Figure 1).

As can be observed in the VSM presented in Figure 1, the hospital's process is as follows: upon arrival, patients would either go directly to an upper floor clinic or the CR area on the first floor. Those who go up to the clinics before registration may be asked by a front-desk staff to go back to the first floor to complete registration. However, others avoid additional travel since the front-desk staff at the clinic can complete registration and check-in processes altogether. Once the registration and check-in processes are complete, patients are asked to take a seat and wait for a nurse to call them. Nurses call patients and direct them to the examination room where physicians will conduct the medical appointment.

In other cases, patients arrive at the CR area where registration and check-in processes are performed (no need to do the check-in at the clinic). Patients then go to the clinic and wait to be called by a nurse to go to the room where the medical appointment will occur.

After being seen by nurses and physicians, patients have two possible paths: check out or be directed to run tests at different locations. Assuming the patient needs additional tests, the patient travels back to the first floor to the $\mathrm{CR}$ area to check-in again fro testing. Once at the CR, they need to register (again) for the lab or X-ray. After the lab/x-ray procedures have been completed, two more paths are open: the patient may leave to return later for test results or go to the clinic and wait to see the nurses and physicians regarding test results during the appointment. Once this meeting is over, patients would then check out and leave until the next appointment.

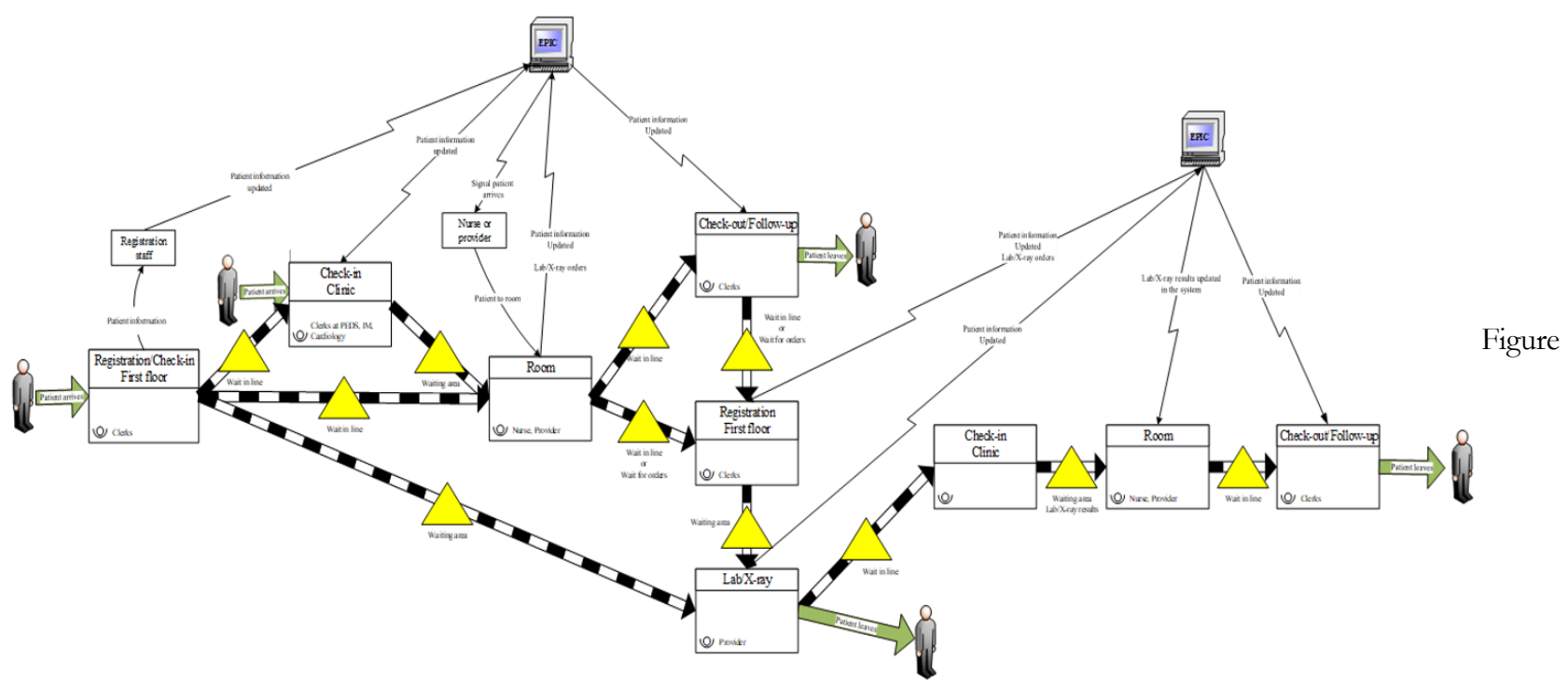

1. VSM of registration and check-in processes (Current state) 
The VSM made clear for hospital stakeholders the number of different and possible paths in the system a patient may take and have to keep in mind every time they go to the hospital for an appointment. In a lean perspective, the first outstanding observation relates to the fact that patients have multiple pathways instead of a single stream. These conditionalities are what precisely create confusion among visitors, whether they are first-time attendants or follow-ups. Consistencies in a service design should be a priority in a lean process since it can help develop a culture for both patients and staff.

Figure 2 depicts value-added and nonvalue-added times from start to end of medical appointment processes in the study's observed clinics. Average times for CR, CR to check-in, check-in, and check-in until a patient is in the clinic room (roomed) were collected for two weeks to assure all shifts were included in the study. The idea was to have a complete picture of the process, especially the CR process, including peak hours and times where the number of patients visits were low. Meeting with the nurse $(\mathrm{RN})$ at the clinic room and waiting and meeting with the physician were collected from the hospital's electronic databases.

The time study conducted at the CR area and the clinics revealed that registration at the hospital was a swift process that could take less than three minutes to complete. On average, registration could take 2.5 minutes; $76 \%$ of the patients who arrive at the CR area waited nearly a minute. A queue could develop only if a staff member is registering a first-time patient, of whom there is no existing information, or there are two or fewer staff members at CR taking patients in.

Based on the information extracted from the time studies, it is possible to affirm that the time spent at CR and CR process performance were not the reasons behind patients' dissatisfaction. Regarding the time for the other processes, it can be seen that check-in and check-out were rapid processes (less than 5 minutes), time with the nurse $(\mathrm{RN})$ is within standards (10-15 minutes) as well as the time with the physician (Doctor). Waiting times (CR to check-in, check-in to room, and waiting for the doctor) are also within standards (5-15 minutes), except for check-in to room in the Cardiology/Pulmonary clinic in which the waiting time is due to forms and documentation that patients have to fill out.

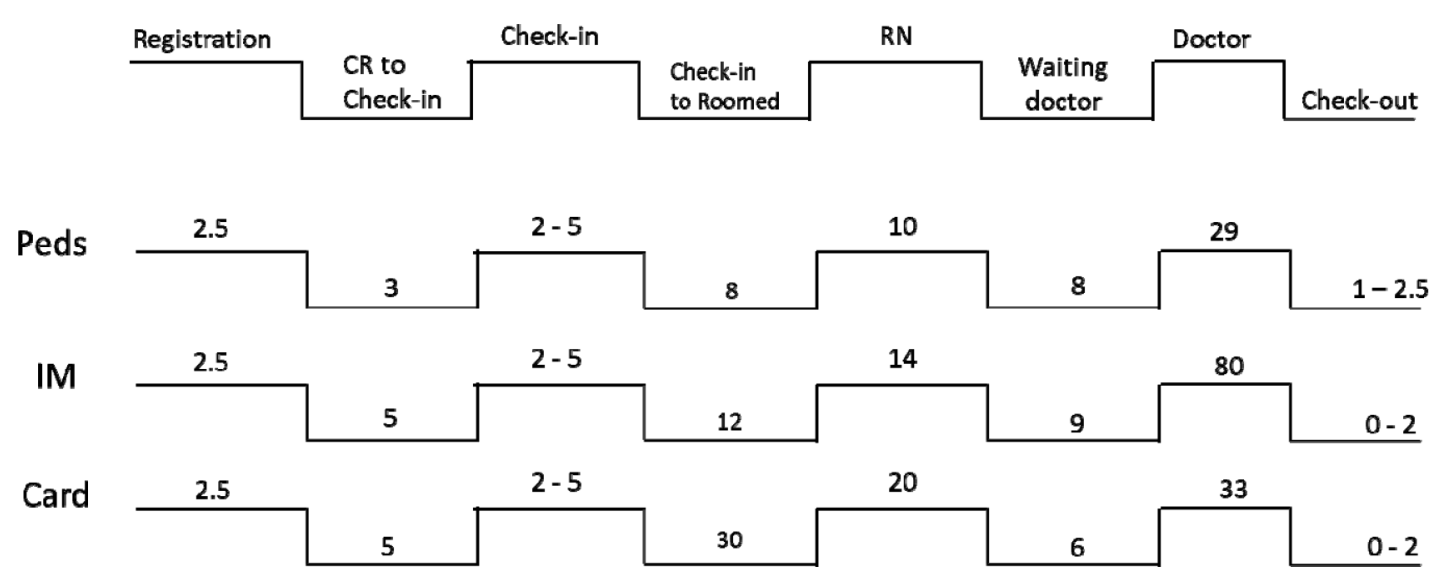

Figure 2. Current process performance times (in minutes)

\subsection{Create Flow and Establish Pull}

As previously mentioned, deficiencies and sources of waste were identified by analyzing the surveys, time studies, and the VSM. Nevertheless, to better understand the problem, it was decided to organize the information and the knowledge gained about the processes using a more intuitive visual thinking tool that allows an understanding of relationships between different topics or areas and aid business analysis. Therefore, a concept map (Figure 3) of the CR process was made. 


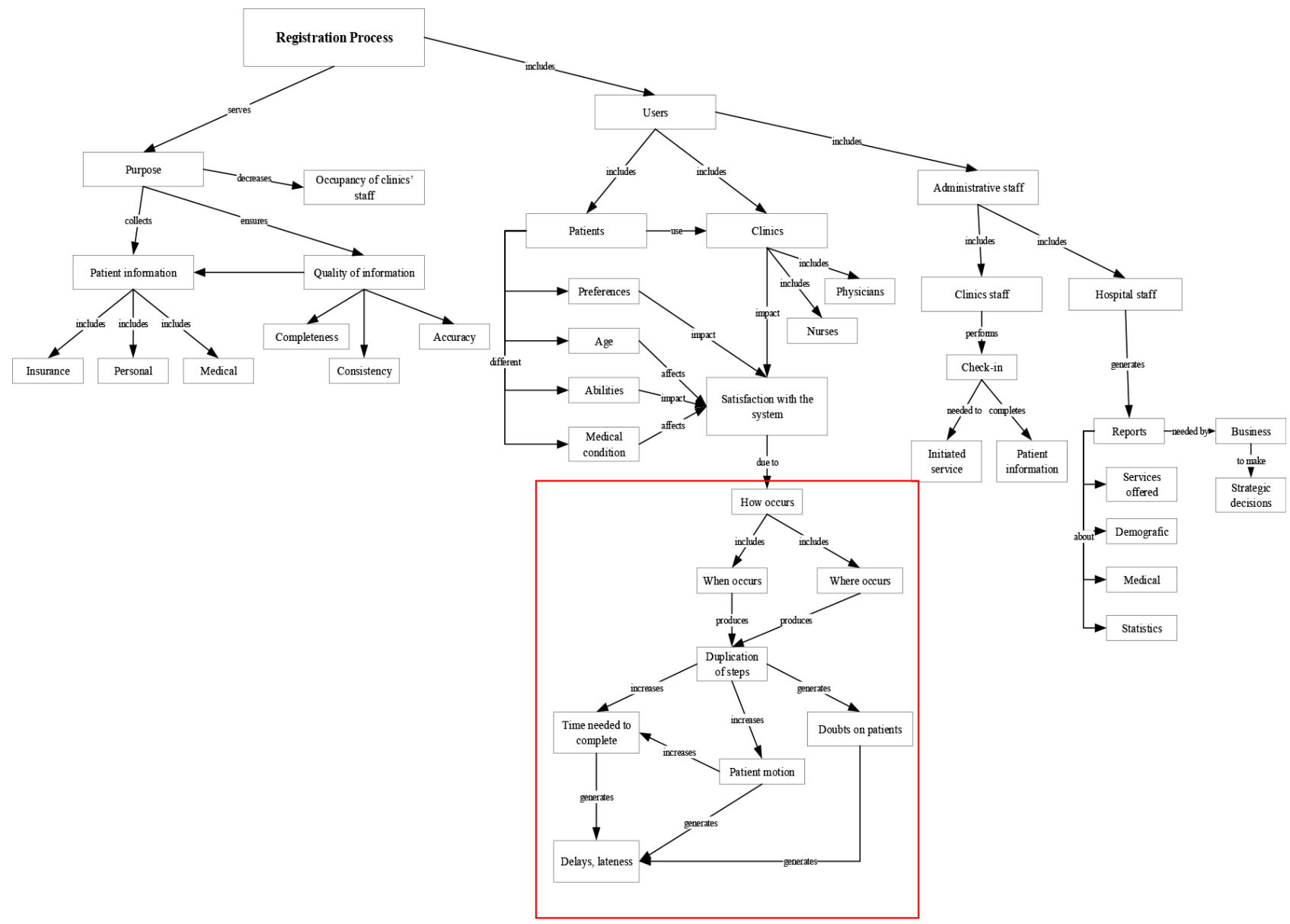

Figure 3. Concept map of the registration process (Current state)

According to the concept map presented in Figure 3, the registration process can be conceptualized by two labels: purpose and users. The red square shows the source of dissatisfaction, which points to three of the major contributors of redundancy expressed here as duplication of steps.

The concept map shows that patients were unsatisfied with how the registration process occurs, especially when and where it occurs. As the new CR process was implemented, patients were confused about where to go. As they figured out where to go, they felt that they were duplicating steps (walking around more) and that the CR process took too much time. As a result, patients felt unsatisfied for doubling overall service time, increasing traveled distances, and generating doubts. Duplication can lead ultimately to delays or latenesses on the patient's end. In that regard, it was generating variability in patient flow at the clinics.

It was confirmed that unnecessary travel created from disorientation in the process and facility provoked registration times to extend beyond normal. Disorientation was deriving from doubts on how the CR functioned. Where and when the registration process should occur were two circumstantial inquiries affecting both patient and staff in the request and delivery of service.

With the information collected, structured, and analyzed until this point of the project, the team (hospital stakeholders and engineering students) started to have clarity on some of the inquiries patients have had: where does the actual registration happen? How can patients get to central registration? What is actually happening at each location (CR, clinic)?. Patient satisfaction is naturally affected by these circumstantial interrogatives. Likewise, hospital stakeholders started to ask themselves where and when should patients opportunely register so that the process turns simple. The engineering team had an additional question: what were the root causes of patient dissatisfaction? It was decided to do a cause and effect diagram (fishbone diagram) presented in Figure 4. 


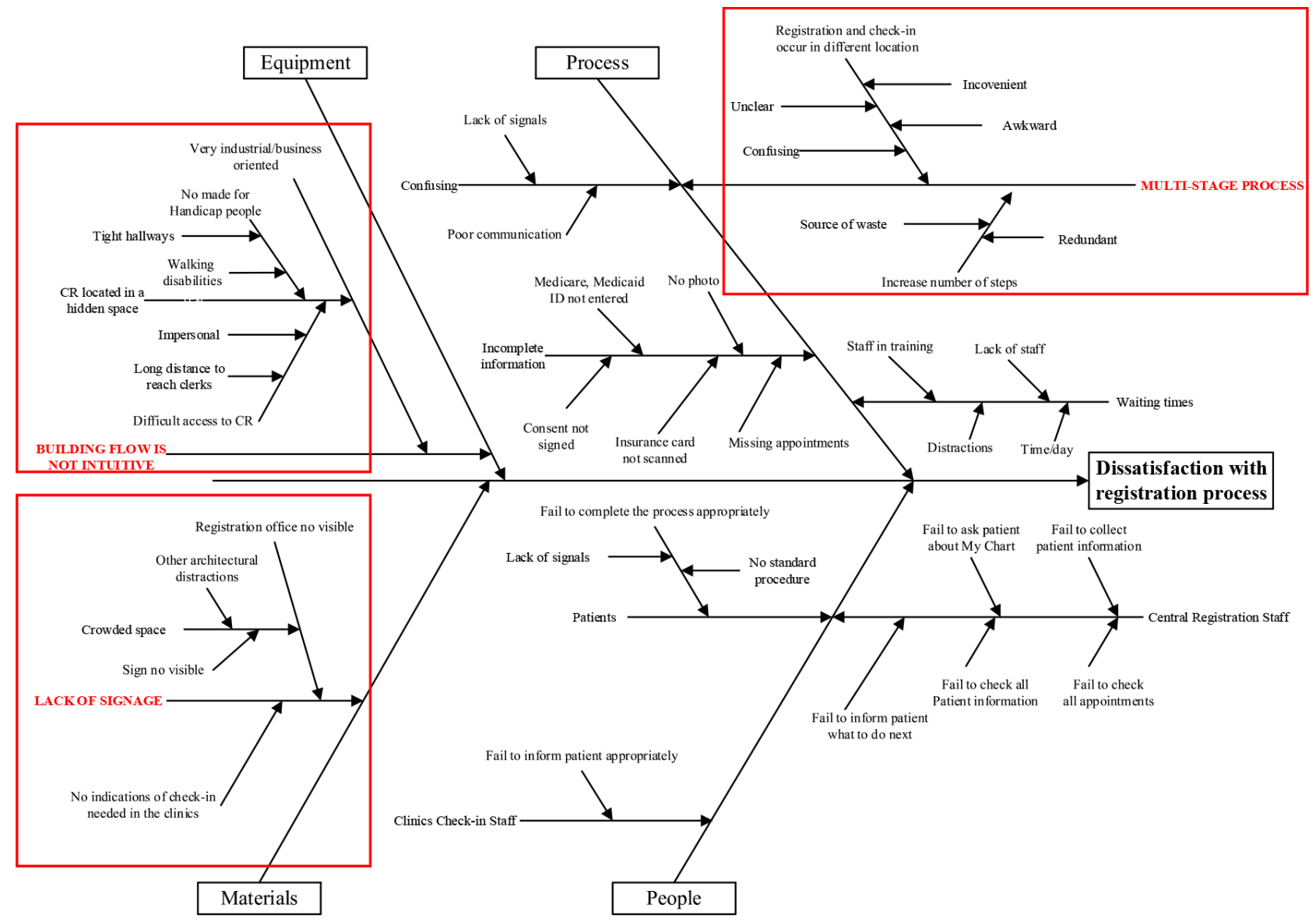

Figure 4. Fishbone diagram of dissatisfaction with the central registration process

The red square in Figure 4 encloses several branches revealing influential causes. The major influencers of the problem were lack of signage, nonintuitive architectural arrangements, and multistage procedures. Several sub causes were extracted from each branch: the process branch, the equipment branch, and the materials branch. The engineering team investigated thoroughly until finding the following root causes: 1) signs indicating CR area location were not visible, 2) registration and check-in are two steps in the process physically apart from each other, 3) CR is located in a hidden space, and 4) poor and puzzling architectural design.

The cause and effect diagram shows that the most notable source of confusion and one of the major influences of the problem was that the building flow was not intuitive. The CR is located in a hidden corner of the building that makes patients go past the elevators that take them to their clinics, causing an awkward, inconvenient, and unclear process. The results and insights collected from the application of the tools mentioned above were analyzed and discussed through group discussions, presentations, and brainstorming sessions. This allowed the team (hospital stakeholders and the engineering team) to create flow and establish pull, defining how the hospital decided to perform the registration and check-in processes.

\subsection{Improvement Recommendations}

Given the results and insights collected from applying the lean tools, the team (hospital stakeholders and the engineering team) moved to make recommendations for improvement. Some of the preliminary recommendations included the possibilities of going back to the prior system and discarding the concept of centralized registration. However, top hospital executives resolved to advocate and maintain the concept of CR, ruling out this recommendation.

Another suggestion explored the idea of implementing airport-like kiosks in a visible space. Still, hospital executives did not approve of this idea due to the difficulties of adjusting to new technology. Also, this idea would cost the hospital greatly; not to mention the cost, and time of software updates. Other ideas were to register in an online 
format, educate patients and staff, supply patients with discernible maps to track trajectory, and installation of visible signage.

Online registration had pros and cons. Although they may lower waiting times and assure privacy, online registration and airport-like kiosks can break much-needed uniformity in the process and add frustrations and other sources of variability. The easier and less disruptive options were to educate patients and staff and put up signage. Education consisted of suggesting specific language on the staff's end and relaxing confusing speech when delivering service. Also, letting patients know ahead of time that registration and check-in are steps distinct from each other helps to ease puzzlement.

The engineering team proposed the rearrangement of the CR area, which would allow better patient flow and visibility of cubicle desks. Figure 5 displays patient motion from the entrance on the upper left to the queueing space and from the queueing space to the service area.

Figure $6(\mathrm{a}, \mathrm{b})$ shows the real-life facility after the rearrangement. Figure $6 \mathrm{a}$ presents the cubicles on the center-right. Figure $6 \mathrm{~b}$ shows the waiting area from a different angle.

However, as stated earlier, the CR area was somewhat hidden in a building with multiple entrances. This detail has affected the intuitive flow to the building, which plays a key role in patient participation (Zhang, Berman \& Verter, 2009) and engagement. Jha et al. (2017) state that patient engagement identifies how a patient interacts with the system's environment. Such an environment can be nourished with effective communication features.

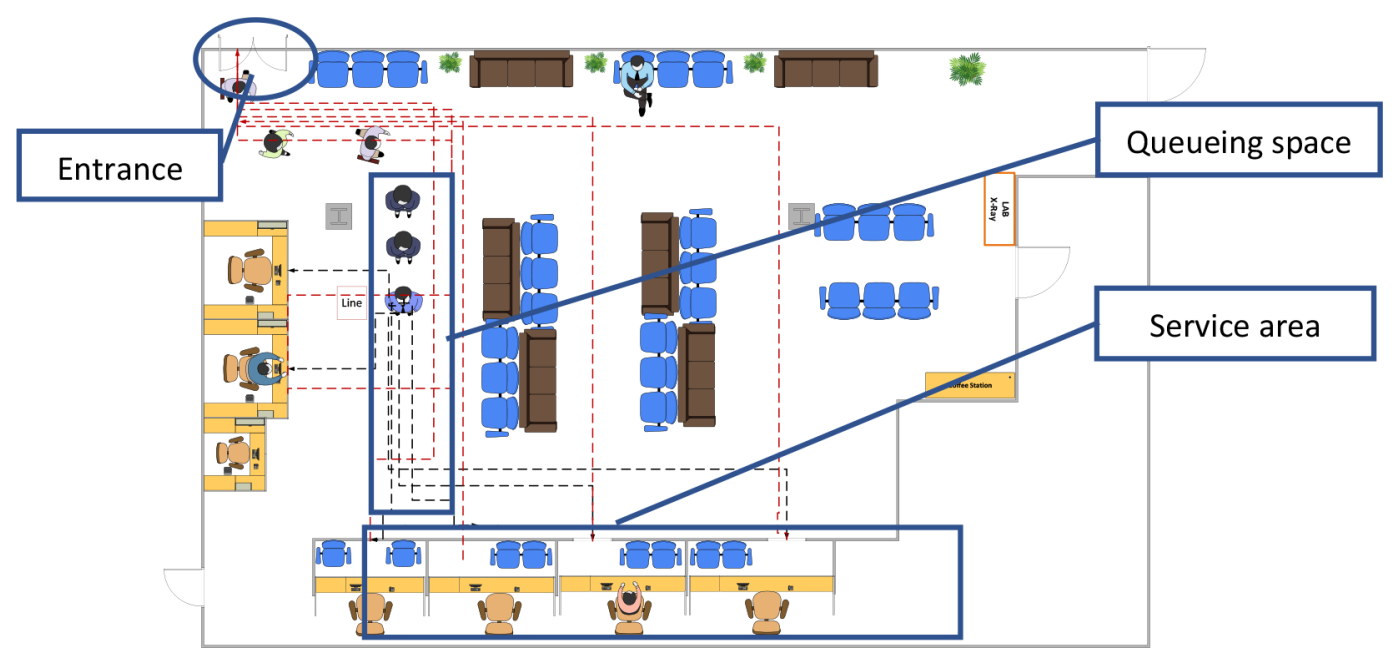

Figure 5. Central registration future design layout
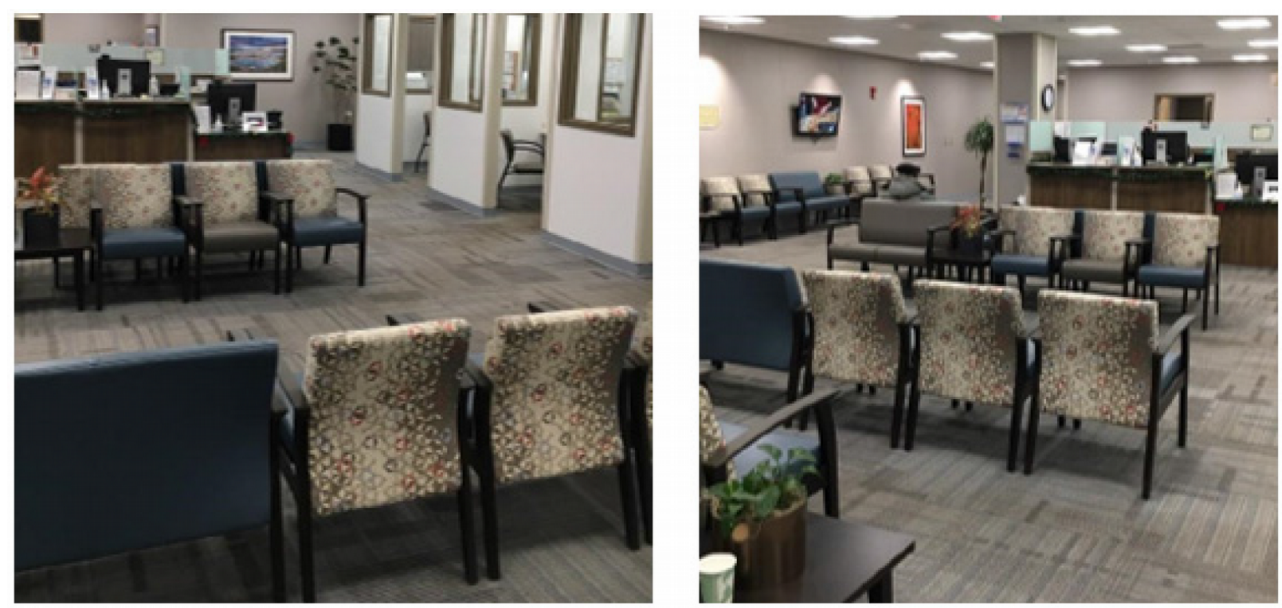

Figure $6(\mathrm{a}, \mathrm{b})$. CR area improved 
As shown in Figure 7 (a, b), the CR area can be easily detoured or skipped from the exterior. To arrive at the lobby, the patient has to move straight ahead and take a right. However, central registration is often bypassed since the elevators are immediately accessible to the left. For wayfinding purposes and per the engineering team's recommendation, the hospital put up a brown sign between the two doors in the back in Figure 7a. The sign says "Registration", preceded by an almost imperceptible (because it is far back) arrow pointing to the right. To make the pathway more intuitive, the hospital originally decided to install a navy-blue wave-like feature (Figure 7a), but it dimmed the light and concealed the brown Registration sign even more. The engineering team suggested that the hospital remove the waves and replace them with arrows on the floor (Figure 7b). The new modification has helped in making clear where the central registration area is located.
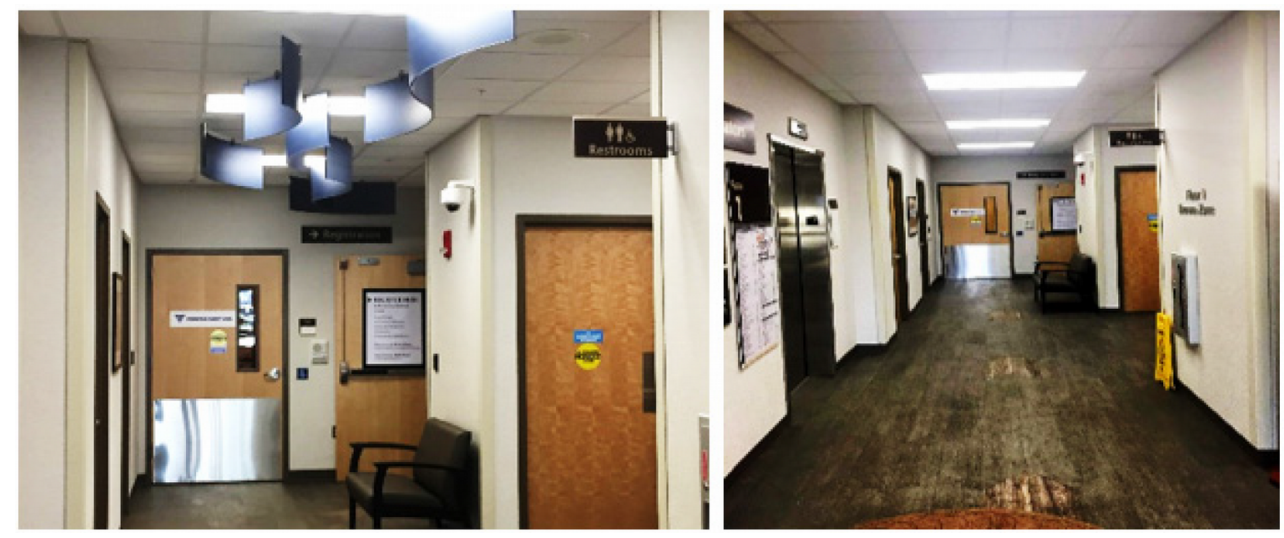

Figure $7(\mathrm{a}, \mathrm{b})$. Central registration entrance before and after

\section{Discussion}

The time study conducted at the Central Registration (CR) area revealed that the hospital's registration process is quick, on average, takes 2.5 minutes. Additionally, $76 \%$ of the patients who arrive at the CR area wait nearly a minute to be registered.

Even though the overall registration process was fairly controlled in duration, the problem started when patients arrived and did not stop by the CR area to be registered. Rather, those who were recurrent attendants would follow the accustomed registration process, the same steps that were followed before the centralization. To be specific, patients would take the elevator to their respective clinics (i.e., IM, P, CP) and present to the front-desk staff, who would unexpectedly tell them to go down to the first floor where CR is located so they can be registered. Patients were not aware of the current procedure the hospital was upholding: registration would be on the first floor, in the lobby, while check-in would be at the clinics. Some patients would even get lost in the entire process and miss their appointments, hence the number of complaints and patient dissatisfaction.

Patient experience is tied to satisfaction. Satisfaction is a competitive advantage over any other organization. Wolf, Niederhauser, Marshburn and LaVela (2014) define patient satisfaction and patient experience. Patient satisfaction is considered a theme of contemporary interest, which continues to be the central goal of many research and improvement projects. Patient experience goes beyond satisfaction, making them two completely separate concepts opening the door for future discussions. The concept of patient satisfaction touches on various elements in a care delivery system, such as organizational cultures (Scott, Mannion, Davies \& Marshall, 2003), individualized care, information, responsiveness, expectations, and perceptions. In this study, patient experience was affected by patients' uninformed expectations and perceptions about the system flow, generating a negative effect on patients' satisfaction.

Some patients were even told not to worry about passing by the CR area and that someone up in the clinic could assist with the registration process, thus creating more confusion for patients and inserting even more variability into the process. Variability is commonly defined as the deviation of a process from its expectation. All processes are subjected to two primary sources of variability: causes inherent to the process and assignable causes that are 
signs of problems in the system (Sower, 2010). It is of great importance to control assignable causes to reduce variability (Djauhari, 2005). When variability is reduced, consistency and predictability are improved, resulting in better productivity, quality, and satisfaction (Shahriari \& Ding, 2011). Roemeling, Land, and Ahaus (2017) argue that a small effort on knowledge dissemination on the roles of variability can have a huge impact on continuous improvement efforts' success.

Lean principles and their application in healthcare should be considered to improve the delivery of care which will improve patient satisfaction. One of the challenges that many teams have faced when applying lean tools in healthcare facilities is getting much-needed support and engagement from principal stakeholders such as medical professionals and administrators. The involvement of stakeholders could make the difference between a success or a failure in the trajectory of a lean project in the healthcare sector (Lindsay, Kumar \& Juleff, 2020; Nino, Claudio, Valladares \& Harris, 2020). In this study, the engineering team worked closely with administrators at the healthcare facility letting them know that their leadership and support made the difference in having impactful outcomes and reaching sustainability of the changes and improvements. In our case, strategies such as empowerment, engagement, and leadership straight from the directors of the areas (central registration and clinics) kept the project moving and simplified implementation. Reach the implementation face wouldn't have been possible without the involvement of nurses and front desk staff in discussion sections and, more importantly, in the decision-making process.

An innovative tool, a concept map, was used in this study to model relationship flows, identify complex relationships among areas and processes, map knowledge, and spot gaps and contradictions in the studied processes. Concept maps are widely used in education (Horton, McConney, Gallo, Woods, Senn \& Hamelin, 1993; Novak, 1990). They are visual representations of information and knowledge. They show concepts and ideas and the relationship among them. Concept maps have expanded to the business world as a powerful strategy since they help see the big picture and present information based on meaningful connections (Dopp, Parisi, Munson \& Lyon, 2019; Trochim \& Kane, 2005). Concept mapping is a group process that makes the tool ideal for stakeholders working together when solving problems (Trochim, Milstein, Wood, Jackson \& Pressler, 2004).

\section{Conclusion}

This project presents a case study on applying lean principles to resolve issues regarding patients' dissatisfaction with registration and check-in processes. The healthcare facility's registration format was negatively affecting patients' experience and therefore deteriorating patients' satisfaction.

Walkthroughs, direct observations, and work samplings facilitated the first two lean principles, 1) define value and 2) map value stream. Understanding the root causes for the gaps in the process (i.e., nonvalue-adding practices, facility arrangement) made possible the following two principles, 3) create flow and 4) establish pull. Finally, there is always room for improvement, so as the fifth principle states, 5) pursue continuous improvement.

The use of the concept map helped the team to visualize the registration and check-in processes holistically. It made it possible to identify the main sources of dissatisfaction and helped to find the root causes more easily.

Hospital stakeholders, including senior managers, supported centralized registration. This alternative reduced staffing and costs, allowed for extended registration hours, reduced turnover among staff members, and minimized registration billing errors. The engineering team suggested signage applications to ensure patient safety, favorable wayfinding strategies, and better time management for both the staff and the patient. The team advised that signs must be readable and free from any physical obstruction.

Lean itself involves a change of culture, a change that can be achieved through education among staff members and clients as needed. It was crucial in this project to orient hospital language with patient flow, so that patients would know that registration and check-in were two detached steps. This proves that small changes can go a long way in improving patient satisfaction in healthcare.

\section{Declaration of Conflicting Interests}

The authors declared no potential conflicts of interest with respect to the research, authorship, and/or publication of this article. 


\section{Funding}

The authors received no financial support for the research, authorship, and/or publication of this article.

\section{References}

Abdu, Y.A., Peter, O., \& Gupta, U.K. (2016). Lean Concepts and Methods: 3P. Int. J. Sci. Res. Comput. Sci. Eng. Inf. Technol, 1(2), 20-24.

Anderson, L.A., Day, K.L., \& Vandenberg, A.E. (2011). Using a concept map as a tool for strategic planning: The Healthy Brain Initiative. Preventing chronic disease, 8(5).

Batbaatar, E., Dorjdagva, J., Luvsannyam, A., Savino, M.M., \& Amenta, P. (2017). Determinants of patient satisfaction: a systematic review. Perspectives in public health, 137(2), 89-101.

https://doi.org/10.1177/1757913916634136

Daultani, Y., Chaudhuri, A., \& Kumar, S. (2015). A decade of lean in healthcare: current state and future directions. Global Business Review, 16(6), 1082-1099. https://doi.org/10.1177/0972150915604520

Deshmukh, S.G., Seth, N., \& Vrat, P. (2005). Service quality models: a review. International Journal of Quality \& Reliability Management, 22(9), 913-949. https://doi.org/10.1108/02656710510625211

Dias, C.R., Pereira, M.R., \& Freire, A.P. (2017). Qualitative review of usability problems in health information systems for radiology. Journal of Biomedical Informatics, 76, 19-33. https://doi.org/10.1016/j.jbi.2017.10.004

Djauhari, M.A. (2005). Improved monitoring of multivariate process variability. Journal of Quality Technology, 37(1), 32-39. https://doi.org/10.1080/00224065.2005.11980298

Dopp, A., Parisi, K., Munson, S., \& Lyon, A. (2019). Integrating implementation and user-centred design strategies to enhance the impact of health services: protocol from a concept mapping study. Health Research Policy and Systems, 17(1), 1. https://doi.org/10.1186/s12961-018-0403-0

Egelhoff, C.J., Podoll, N., \& Tarhini, K. (2010). Work in progress-A Concept Map for Mechanics of Materials. Paper presented at the 2010 IEEE Frontiers in Education Conference (FIE). https://doi.org/10.1109/FIE.2010.5673352

Faezipour, M., \& Ferreira, S. (2013). A System Dynamics Perspective of Patient Satisfaction in Healthcare. Paper presented at the CSER. https://doi.org/10.1016/j.procs.2013.01.016

Fatima, T., Malik-Shahab, A., \& Shabbir, A. (2018). Hospital healthcare service quality, patient satisfaction and loyalty: An investigation in context of private healthcare systems. International Journal of Quality \& Reliability Management, 35(6), 1195-1214. https://doi.org/10.1108/IJQRM-02-2017-0031

Faulkner, B. (2013). Applying lean management principles to the creation of a postpartum hemorrhage care bundle. Nursing for women's health, 17(5), 400-411. https:// doi.org/10.1111/1751-486X.12063

Gershenson, J., \& Pavnaskar, S. (2003). Eight basic lean product development tools. Paper presented at the DS 31: Proceedings of ICED 03, the 14th International Conference on Engineering Design. Stockholm.

Giordano, L.A., Elliott, M.N., Goldstein, E., Lehrman, W.G., \& Spencer, P.A. (2010). Development, Implementation, and Public Reporting of the HCAHPS Survey. Medical Care Research and Revien, 67(1), 27-37. https://doi.org/10.1177/1077558709341065

Hallam, C.R., \& Contreras, C. (2018). Lean healthcare: scale, scope and sustainability. International journal of health care quality assurance. https://doi.org/10.1108/IJHCQA-02-2017-0023

Hicks, C., McGovern, T., Prior, G., \& Smith, I. (2015). Applying lean principles to the design of healthcare facilities. International Journal of Production Economics, 170, 677-686. https://doi.org/10.1016/j.ijpe.2015.05.029

Horton, P.B., McConney, A.A., Gallo, M., Woods, A.L., Senn, G.J., \& Hamelin, D. (1993). An investigation of the effectiveness of concept mapping as an instructional tool. Science Education, 77(1), 95-111.

https://doi.org/10.1002/sce.3730770107

Jha, D., Frye, A.K., \& Schlimgen, J. (2017). Evaluating variables of patient experience and the correlation with design. Patient Experience Journal, 4(1), 33-45. https://doi.org/10.35680/2372-0247.1176 
Kadarova, J., \& Demecko, M. (2016). New approaches in lean management. Procedia Economics and Finance, 39(1), 11-16. https:// doi.org/10.1016/S2212-5671(16)30234-9

Leviner, S. (2020). Patient flow within hospitals: A conceptual model. Nursing Science Quarterly, 33(1), $29-34$. https://doi.org/10.1177/0894318419881981

Lindsay, C.F., Kumar, M., \& Juleff, L. (2020). Operationalising lean in healthcare: the impact of professionalism. Production Planning \& Control, 31(8), 629-643. https:// doi.org/10.1080/09537287.2019.1668577

Lot, L.T., Sarantopoulos, A., Min, L.L., Perales, S.R., Boin, I.D.F.S.F., \& Ataide, E.C. (2018). Using Lean tools to reduce patient waiting time. Leadership in health services. https://doi.org/10.1108/LHS-03-2018-0016

MacAllister, L., Zimring, C., \& Ryherd, E. (2016). Environmental variables that influence patient satisfaction: A review of the literature. HERD: Health Environments Research \& Design Journal, 10(1), 155-169. https://doi.org/10.1177/1937586716660825

Machado, V.C., \& Leitner, U. (2010). Lean tools and lean transformation process in health care. International Journal of Management Science and Engineering Management, 5(5), 383-392. https://doi.org/10.1080/17509653.2010.10671129

Maqbool, T., Raju, S., \& In, E. (2016). Importance of patient-centred signage and navigation guide in an orthopaedic and plastics clinic. BMJ Open Quality, 5(1). https://doi.org/10.1136/bmjquality.u209473.w3887

Martin-Escalona, I., Barcelo-Arroyo, F., \& Zola, E. (2013). The introduction of a topic on accessibility in several engineering degrees. Paper presented at the 2013 IEEE Global Engineering Education Conference (EDUCON). https://doi.org/10.1109/EduCon.2013.6530177

Mathew, B., Narayanan, R., \& Mittal, A. (2019). A review of the perception of healthcare quality among patients and employees. Indian Journal of Public Health Research \& Development, 10(9), 452-456.

https://doi.org/10.5958/0976-5506.2019.02469.0

Mazurenko, O., Collum, T., Ferdinand, A., \& Menachemi, N. (2017). Predictors of hospital patient satisfaction as measured by HCAHPS: a systematic review. Journal of Healthcare Management, 62(4), 272-283. https://doi.org/10.1097/JHM-D-15-00050

Meesala, A., \& Paul, J. (2018). Service quality, consumer satisfaction and loyalty in hospitals: Thinking for the future. Journal of Retailing and Consumer Services, 40, 261-269. https://doi.org/10.1016/j.jretconser.2016.10.011

Moretta-Tartaglione, A., Cavacece, Y., Cassia, F., \& Russo, G. (2018). The excellence of patient-centered healthcare: Investigating the links between empowerment, co-creation and satisfaction. The TQM Journal, 30(2), 153-167. https://doi.org/10.1108/TQM-11-2017-0138

Nino, V., Claudio, D., Valladares, L., \& Harris, S. (2020). An Enhanced Kaizen Event in a Sterile Processing Department of a Rural Hospital: A Case Study. International Journal of Environmental Research and Public Health, 17(23), 8748. Available at: https://www.mdpi.com/1660-4601/17/23/8748

Novak, J.D. (1990). Concept maps and Vee diagrams: two metacognitive tools to facilitate meaningful learning. Instructional Science, 19(1), 29-52. https://doi.org/10.1007/BF00377984

Nunnally, M.E., \& Bitan, Y. (2014). Ad hoc signage in a new hospital-a useful social tool and cognitive artifact. Paper presented at the Proceedings of the International Symposium on Human Factors and Ergonomics in Health Care. https://doi.org/10.1177/2327857914031015

Oliver, R.L. (1993). A Conceptual Model of Service Quality and Service Satisfaction: Comparative Goals, Different Concepts. Advances in Service Marketing and Management, 2, 65-85. Available at: https://ci.nii.ac.jp/naid/10031116648/en/

Parasuraman, A., Zeithaml, V.A., \& Berry, L.L. (1985). A Conceptual Model of Service Quality and Its Implications for Future Research. Journal of Marketing, 49(4), 41-50. https://doi.org/10.1177/002224298504900403

Robinson, S., Radnor, Z.J., Burgess, N., \& Worthington, C. (2012). SimLean: Utilising simulation in the implementation of lean in healthcare. European Journal of Operational Research, 219(1), 188-197.

https://doi.org/10.1016/j.ejor.2011.12.029 
Roemeling, O., Land, M., \& Ahaus, K. (2017). Does lean cure variability in health care? International Journal of Operations \& Production Management, 37(9), 1229-1245. https://doi.org/10.1108/IJOPM-07-2015-0452

Scott, T., Mannion, R., Davies, H.T., \& Marshall, M.N. (2003). Implementing culture change in health care: theory and practice. International journal for quality in health care, 15(2), 111-118. https://doi.org/10.1093/intqhc/mzg021

Series, I.I. (2005). Going Lean in Health Care. Cambridge, MA: Inst. for Healthcare Improvement.

Shahriari, K., \& Ding, F. (2011). Process variability and inherent efficiency enhancement in industrial processes: Two case studies in pulp and paper industry. Paper presented at the 2011 IEEE International Conference on Control Applications (CCA). https://doi.org/10.1109/CCA.2011.6044512

Sobek, D.K. (2011). Lean Healthcare Implementation: Critical Success Factors. IIE Annual Conference. Proceedings (1-8).

Solaimani, S., van der Veen J., Sobek, I.I.D.K., Gulyaz, E., \& Venugopal, V. (2019). On the application of Lean principles and practices to innovation management: A systematic review. The TQM Journal, 31(6), 1064-1092. https://doi.org/10.1108/TQM-12-2018-0208

Sower, V. (2010). Essentials of quality with cases and experiential exercises: John Wiley \& Sons.

Spagnol, G.S., Min, L.L., \& Newbold, D. (2013). Lean principles in Healthcare: an overview of challenges and improvements. IFAC Proceedings Volumes, 46(24), 229-234. https://doi.org/10.3182/20130911-3-BR-3021.00035

Suess, C., \& Mody, M. (2018). The influence of hospitable design and service on patient responses. The Service Industries Journal, 38(1-2), 127-147. https://doi.org/10.1080/02642069.2017.1385773

Trochim, W., \& Kane, M. (2005). Concept mapping: an introduction to structured conceptualization in health care. International journal for quality in health care, 17(3), 187-191. https://doi.org/10.1093/intqhe/mzi038

Trochim, W., Milstein, B., Wood, B., Jackson, S., \& Pressler, V. (2004). Setting Objectives for Community and Systems Change: An Application of Concept Mapping for Planning a Statewide Health Improvement Initiative. Health Promotion Practice, 5(1), 8-19. https://doi.org/10.1177/1524839903258020

Wickramasinghe, N. (2014). Lean principles for healthcare. In Lean Thinking For Healthcare (3-11). Springer. https://doi.org/10.1007/978-1-4614-8036-5_1

Wild, D.M.G., Kwon, N., Dutta, S., Tessier-Sherman, B., Woddor, N., Sipsma, H.L. et al. (2011). Who's Behind an HCAHPS Score? The joint commission journal on quality and patient safety, 37(10), 461-AP463. https://doi.org/10.1016/S1553-7250(11)37059-6

Wolf, J.A., Niederhauser, V., Marshburn, D., \& LaVela, S.L. (2014). Defining patient experience. Patient Experience Journal, 1(1), 7-19. https://doi.org/10.35680/2372-0247.1000

Womack, J., \& Jones, D., \& Roos, D. (1990). The Machine That Changed the World. New York: Rawson Associates.

Yellen, E., Davis, G.C., \& Ricard, R. (2002). The measurement of patient satisfaction. Journal of Nursing Care Quality, 16(4), 23-29. https://doi.org/10.1097/00001786-200207000-00005

Zhang, Y., Berman, O., \& Verter, V. (2009). Incorporating congestion in preventive healthcare facility network design. European Journal of Operational Research, 198(3), 922-935. https://doi.org/10.1016/j.ejor.2008.10.037

Journal of Industrial Engineering and Management, 2021 (www.jiem.org)

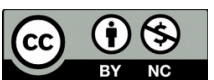

Article's contents are provided on an Attribution-Non Commercial 4.0 Creative commons International License. Readers are allowed to copy, distribute and communicate article's contents, provided the author's and Journal of Industrial Engineering and Management's names are included. It must not be used for commercial purposes. To see the complete license contents, please visit https://creativecommons.org/licenses/by-nc/4.0/. 